\section{Critique of the DSM-IV operational diagnostic criteria for schizophrenia}

MARIO MAJ

The DSM-IV operational diagnostic criteria for schizophrenia (American Psychiatric Association, 1994) are being used extensively in several countries for clinical, research and educational purposes.

Unlike their older predecessors - the $\mathrm{St}$ Louis criteria (Feighner et $a l, 1972$ ) and the Research Diagnostic Criteria (Spitzer et al, 1978) - the DSM-IV criteria are being used not only by experienced psychiatrists with an in-depth knowledge of psychopathology (as a quick reference for the reliable selection of individuals for research), but also by students and residents, who often first approach the concept of schizophrenia through these criteria. For some of these people, the DSM-IV will remain the only source of knowledge about schizophrenia.

It is time, after three years of extensive use for several purposes, for a critical evaluation of the DSM-IV criteria. If they have major limitations, this should be acknowledged, and possible remedies should be discussed. The present paper represents a first step in this direction.

\section{OVERVIEW OF THE CRITERIA}

The DSM-IV definition of schizophrenia consists of a symptomatological criterion, a chronological criterion, a functional criterion and some exclusion criteria.

The symptomatological criterion requires the presence of at least two of the following symptoms: delusions, hallucinations, disorganised speech (e.g. frequent derailment or incoherence), grossly disorganised or catatonic behaviour, and negative symptoms (affective flattening, alogia or avolition). A bizarre delusion is sufficient for the diagnosis, even if it is the only symptom. The same applies to hallucinations made up of commenting voices or of two or more voices conversing with each other.

The chronological criterion demands that the above symptoms be present for at least one month (or less if successfully treated) and that "continuous signs of the disturbance" be present for at least six months. This six-month period may include a prodromal and/or a residual phase, marked by only negative symptoms or by two or more of the above-mentioned symptoms present in an attenuated form (e.g. odd beliefs or unusual perceptual experiences).

The functional criterion requires that "for a significant portion of the time since the onset of the disturbance" there is a marked impairment of functioning in one or more areas such as work, interpersonal relations or self-care.

The exclusion criteria are that mood and schizoaffective disorders have been ruled out, and that the disturbance is not due to the direct physiological effects of a substance (drug of abuse or medication) or a general medical condition. Elsewhere in the manual it is specified that the diagnosis of schizophrenia is excluded if the syndrome can be ascribed to a neurological disorder (including central nervous system infections), an endocrine or metabolic condition, a fluid or electrolyte imbalance, a renal or heparic disease or an auroimmune disorder.

\section{ANALYSIS OF THE CRITERIA}

Each of the criteria mentioned above raises several problems.

The main problem with the symptomatological criterion is that it does not characterise schizophrenia as a syndrome. It may well be true that the definition of schizophrenia must be polythetic rather than monothetic, since there is no single symptom that is pathognomonic of the disorder (Andreasen, 1987), but at least the clustering of symptoms should be characteristic. Unfortunately, the DSM-IV symptomatological criterion does not identify such a characteristic clustering. In fact, that criterion can be fulfilled by several cases of mania (disorganised speech plus grossly disorganised behaviour), major depression with psychotic features (alogia plus delusions), dementia (grossly disorganised behaviour plus hallucinations) and delirium (disorganised speech plus hallucinations). Two independent groups have found that a psychopathological structure consisting of psychotic, negative and disorganised dimensions is common to schizophrenia and bipolar disorder (Klimidis et al, 1993; Maziade et al, 1995).

A second problem with the symptomatological criterion is that the five groups of symptoms it mentions (delusions, hallucinations, disorganised speech, grossly disorganised or catatonic behaviour and negative symptoms) are all given the same weight in the diagnostic process. The combination of grossly disorganised behaviour and alogia qualifies for the diagnosis of schizophrenia as well as the co-occurrence of delusions and hallucinations. This is likely to create difficulties in community mental health settings, where the issue of the differential diagnosis between mental illness and social deviance often arises. Should any vagrant who displays grossly disorganised behaviour (for example, by appearing markedly dishevelled or dressing in an unusual manner) and poverty of speech be diagnosed as having schizophrenia?

A third issue raised by the symptomatological criterion concerns the threshold above which the clinical manifestations listed in the criterion should be regarded as symptoms. Andreasen \& Flaum (1991) previously commented on the change in the base rates of psychotic and negative symptoms according to the severity threshold that is selected, but this issue is probably even more sensitive for disorganised speech and disorganised behaviour, which are likely to be more common in the general population. The decision whether or not speech or behaviour disturbances should be regarded as symptoms in an individual case is completely left to the subjective judgement of the clinician. The reliability of this judgement is likely to be poor, and to decrease significantly as one moves from an in-patient to a community setting.

Finally, the concept of bizarre delusions has been kept in the DSM-IV definition of schizophrenia despite the empirical evidence of the poor interrater reliability in the evaluation of a delusion as bizarre or non-bizarre (Kendler et al, 1983, Flaum et $a l, 1991)$. This is of special concern since, if a delusion is regarded as bizarre, it qualifies 
for the diagnosis of schizophrenia even if it is the only symptom.

Within the chronological criterion, the requirement of a continuity of the disturbance for at least six months raises the wellknown issues (Keith \& Matthews, 1994) of tautological prediction of outcome (the longer one has an illness, the more likely one is to have it for a long time) and divergence from the ICD-10 (World Health Organization, 1992). What is of special concern, however, is the doubtful reliability of a rule that: (a) requires the retrospective assessment not only of the presence in the past, but also of the date of onset of several clinical manifestations; (b) implies a subjective judgement about whether these retrospectively identified manifestations are indeed 'prodromal' to the active-phase syndrome (i.e. are actually part of the 'disturbance'); and (c) does not involve only proper symptoms, but also such impalpable features as odd beliefs and unusual perceptual experiences. (What is an unusual perceptual experience? How common are these experiences in the general population? What is the threshold above which these experiences should be regarded as pathological?) An empirical study (Andreasen \& Flaum, 1994) assessing the test-retest reliability of prodromal unusual perceptual experiences has found an intraclass $r$ of zero.

The functional criterion, as it appears in the DSM-IV, is the outcome of the laborious attempt to introduce in the definition of schizophrenia the concept of functional decline (again, absent in the ICD-10), without implying that this decline is irreversible. However, in its present form, the criterion is very unclear. What is the meaning of the formula "for a significant portion of the time since the onset of the disturbance"? If the subject's social functioning is impaired only during the onemonth period in which he is actively psychotic, is the criterion fulfilled? Should we accept one month as a "significant portion of time", or should we require the persistence of social impairment for some additional time after the remission of psychotic symptoms? Again, the decision is up to the individual clinician, and the criterion is applied very inconsistently.

In the exclusion criteria, the most questionable aspect is the incorporation of an aetiological component, as the diagnosis of schizophrenia is not allowed if the syndrome can be ascribed, for instance, to a central nervous system infection. Indeed, if schizophrenia is presented as a syndrome (not as a disease), and this is the case in the DSM-IV, its concept should not be demarcated by an aetiological element. Otherwise, if in the future it will be possible to demonstrate that some cases currently receiving the diagnosis of schizophrenia are due, for instance, to a viral infection (as suggested by some contemporary research), the diagnosis will have to be changed in those cases. The mere fact that aetiology has been identified will imply that the condition is not schizophrenia!

\section{DOES THE SCHIZOPHRENIC SYNDROME HAVE A CHARACTER?}

What emerges from the above analysis of the DSM-IV diagnostic criteria for schizophrenia is that they lack an underlying paradigm. There are residua of the classical Kraepelinian, Bleulerian and Schneiderian paradigms (respectively, in the provision of a chronological and a functional criterion, in the reference to disorganised speech and affective flattening, and in the inclusion of some first-rank symptoms in the symptomatological criterion). However, none of those paradigms is actually endorsed (not the Kraepelinian one, since a deteriorating course is not finally required; not the Bleulerian one, since the diagnosis of schizophrenia can be made even in the absence of all Bleuler's fundamental symptoms; not the Schneiderian one, since even if all first-rank symptoms are present, but a full affective syndrome co-exists, the diagnosis will be of affective disorder). The positive-negative dichotomy, which has emerged during the past two decades as a possible new paradigm, is mentioned at the beginning of the DSM-IV chapter on schizophrenia ("the essential features of schizophrenia are a mixture of characteristic signs and symptoms, both positive and negative"), but this paradigm also is not finally endorsed in the criteria (in fact, according to the DSM-IV definition, the diagnosis of schizophrenia is possible even in the complete absence of both positive and negative symptoms, if disorganised speech and grossly disorganised behaviour are both present).

The DSM-IV diagnosis of schizophrenia finally becomes a diagnosis by exclusion. The symptomatological, chronological and functional criteria, taken together, do not characterise schizophrenia as a syndrome (all of them may be fulfilled by several cases of dementia, major depression, or bipolar disorder), so that the exclusion criteria become decisive for the diagnosis. Should the schizophrenic syndrome be really diagnosed by exclusion? Does this syndrome not have a character? Or are the DSM-IV criteria simply unable to convey a character that does exist?

There are at least four possible answers to these questions:

(a) What we currently call schizophrenia is merely a heterogeneous group of nonaffective psychotic syndromes whose aetiology is not known (in this case, the DSM-IV would be right in suggesting a diagnosis by exclusion, but the issue would arise whether it is not appropriate to replace the term schizophrenia by a more neutral expression, such as 'idiopathic psychoses' or 'primary psychoses')

(b) The schizophrenic syndrome does have a character, but its essence cannot be translated into operational terms (for instance, this essence does not lie in a constellation of symptoms, but in a "mode of being" of the subject (Kraus, 1994), so that the trained psychiatrist, in making the diagnosis of schizophrenia, does not rely only on the identification of some symptoms, but also on a holistic impression of the subject, which operational criteria are unable to communicate)

(c) The schizophrenic syndrome does have a character, but the DSM-IV criteria fail to catch one or more clinical aspects that are essential for the diagnosis (for instance, there may be something more in Bleuler's fundamental symptoms than in negative symptoms as they are currently conceptualised and defined)

(d) The schizophrenic syndrome does have a character, and all its essential elements are present in the DSM-IV definition, but either these elements are not described in sufficient detail (in its skeletal essentiality, the DSM-IV criteria may be easy to remember, but may fail to qualify sufficiently the individual clinical features of schizophrenia) or the clustering of symptoms that characterises schizophrenia is not appropriately identified.

\section{FUTURE PERSPECTIVES}

One could argue that we have come to a critical point in which it is difficult to 
discern whether the operational approach is disclosing the intrinsic weakness of the concept of schizophrenia (showing that the schizophrenic syndrome does not have a character and can be defined only by exclusion) or whether the case of schizophrenia is bringing to light the intrinsic limitations of the operational approach (showing that this approach is unable to convey the clinical flavour of such a complex syndrome). In other terms, there may be, beyond the individual phenomena, a "psychological whole" (Jaspers, 1963) in schizophrenia, that the operational approach fails to grasp, or such a psychological whole may simply be an illusion, that the operational approach unveils.

There is, however, a further possibiliry: that the resources of the operational approach have not been fully exploited in the case of schizophrenia. For instance, if such eminent clinicians and scholars as Bleuler, Kretschmer and Biswanger have regarded autism as one of the key features of schizophrenia, then the recent efforts to define reliably this clinical aspect (Gündel \& Rudolf, 1993) and to grasp the nature of the basic relational deficit shared by individuals with schizophrenia and schizophrenic spectrum disorders (Parnas \& Bovet, 1991) deserve attention. On the other hand, it is possible that the form and content of the subjective experiences of individuals who are diagnosed as having schizophrenia require a more in-depth investigation and characterisation, reversing the recent process of reduction of psychotic phenomena to their smallest common denominator, of which the DSM-IV laconic formulation is the outcome.

A renaissance of psychopathological research, focusing on the above issues, should be, in my opinion, encouraged.

MARIO MAJ. MD, Depar tment of Psychiatry, University of Naples, Italy

Correspondence: Professor Mario Maj. Department of Psychiatry. University of Naples. Largo Madonna delle Grazie. 80138 Naples, Italy

(First received I5 July 1997, final revision I December 1997, accepted 5 December 1997)

Meanwhile, the DSM-IV operational diagnostic criteria for schizophrenia should be used sparely, if at all, for educational purposes. If the few words composing the DSM-IV definition will probably evoke, in the mind of expert clinicians, the complex picture that they have learnt to recognise along the years, the same cannot be expected for students and residents. For these people, those few words will remain what they are, and the risk of misunderstanding and oversimplification will be very high.

\section{ACKNOWLEDGEMENT}

I thank Dr Paola Bucci for her help with the manu script.

\section{REFERENCES}

American Psychiatric Association (1994) Diognostic and Statistical Manual of Mental Disorders (4th edn) (DSM-IV). Washington, DC: APA

Andreasen, N. C. (1987) The diagnosis of schizophrenia. Schizophrenio Bulletin. 13, 9-22.

- Flaum, M. (1991) Schizophrenia: the characteristic symptoms. Schizophrenia Bulletin. 17. $27-49$.

- 2 (1994) Characteristic symptoms of schizophrenia. In DSM-IV Sourcebook. Vol. I (eds T. A. Widiger, A. J. Frances, H. A. Pincus. et al). pp. 351-380. Washington. DC: APA

Feighner, J. P., Robins, E., Guze, S. B., et at (1972) Diagnostic criteria for use in psychiatric research. Archives of Generol Psychioury, 26. 57-63.
Flaum, M., Arndt, S. \& Andreasen, N. C. (1991) Reliability of bizarre delusions. Comprehensive Psychiotry, 32. $59-65$

Gündel, H. \& Rudolf, G. A. E. (1993) Schizophrenic autism. 2. Proposal for a nomothetic definition Psychopathology. 26. 304-312.

Jaspers, K. (1963) General Psychopathology Chicago. IL: University of Chicago Press.

Keith, S. J. \& Matthews, S. M. (1994) The diagnosis of schizophrenia: a review of onset and duration issues. In DSM-IV Sourcebook. Vol. I (eds T. A.Widiger, A. J. Frances, H. A. Pincus. et al). pp. 393-417. Washington, DC: APA.

Kendler, K. S., Glazer, W. M. \& Morgenstern, H. (1983) Dimensions of delusional experience. American Journol of Psychioury, 40, 466-469.

Klimidis, S., Stuart, G.W., Minas, I. H., et of (1993) Positive and negative symptoms in the psychoses: re-analysis of published SAPS and SANS globat ratings. Schizophrenia Research, 9.11-18.

Kraus, A. (1994) Phenomenological and criteriologica diagnosis. Different or complementary? In Philosophical Perspectives on Psychiatric Diagnostic Clossification (eds 1. Z. Sadler. D. P.Wiggins \& M. A. Schwartz), pp. 148-160. Baltimore. MD: Johns Hopkins University Press.

Maziade, M., Roy, M.-A., Martinez, M., et al (1995) Negative, psychoticism, and disorganized dimensions in patients with familial schizophrenia or bipolar disorder: continuity and discontinuity between the major psychoses. American journol of Psychiotry. 152. $1458-1463$

Parnas, I. Bovet, P. (I99I) Autism in schizophrenia revisited. Comprehensive Psychotry. 32. 7-21.

Spitzer, R. L., Endicott, J. Robins, E. (1978) Research Diagnostic Criterio (RDC) for a Selected Group of Functional Disorders (3rd edn). New York: New York State Psychiatric Institute.

World Health Organization (1992) The ICD-10 Clossification of Mental and Behovioural Disorders. Clinical Descriptions and Diognostic Gurdelines. Geneva: WHO 